\title{
Design and Implementation of Corporation Robots
}

\author{
Saleh Faraj Mansour Sulaiman ${ }^{1, *}$, Abdulrahman A. A. Emhemed ${ }^{2,3}$ \\ ${ }^{1}$ Higher Institute of Engineering Technology, Bani Walid, Libya \\ ${ }^{2}$ College of Technical Sciences, Bani Walid, Libya \\ ${ }^{3}$ Engineering and Information Technology Research Centre, Bani Walid, Libya
}

Copyright $\subseteq 2018$ by authors, all rights reserved. Authors agree that this article remains permanently open access under the terms of the Creative Commons Attribution License 4.0 International License

\begin{abstract}
Corporation robots basically consist of two robots that collaborate together to perform shared goals. Single robot is not suitable to handle heavy load and cannot handle long size load. Therefore, the corporation robots system is introduced to overcome with these limitations. This paper is mainly concerned on the design and construction of the corporation robots that can follow line and carry load. The PIC16F877A is used as microcontroller brain, while the circuit will be built and connected to IR sensors and motors. The corporation robots follow the line and work together as leader and slave to carry load from one point to another point. The experimental results achieved good performance for the robots movement regarding accurate line following and smooth handling the load.
\end{abstract}

Keywords Corporation Robots, Leader Follower, Line Follower, DC Motor

\section{Introduction}

Robots are electromechanical machines having ability to perform tasks or actions on some given electronic programming. Line follower corporation robots are mobile robots having ability to work together with accurate behavior. Various studies have been conducted since the word "robot" was brightened. Line follower robots have been used in industrial logistics. Leader and slave following line robots were designed by many scientists due to its low cost and ability to do more than one heavy-duty. An ideal application for groups of robots working together, like a society of insect, can accomplish the same mission that one robot. Using simpler mobile robots doing specific task, is less expensive, more reliable and it can reach the same aims of one robots. Some examples of applications are in manufacturing, medicine, space exploration and home. The complexity of many environments or works may require a mixture of robotic capabilities that is too expensive to design into a single robot. Additionally, time constraints may require the use of multiple robots working simultaneously on different aspects of the mission in order to successfully accomplish the objective [1]. Two small scale vehicles were developed by university technology Malaysia at 2007. One is the leading vehicle and another is the following vehicle. The following vehicle could follow the leading vehicle in straight line. Both vehicles utilize PIC 18F454 microcontroller (MCU) as the "brain" for both vehicles. The following vehicle utilizes ultrasonic sensor (R40-16 \& T40-16) to detect and measure the distance between the leading and the following vehicle. When the ultrasonic sensor sends a wave with certain frequency and received it back through reflection after hit the obstacle, the information was sent to the PIC microcontroller. The Microcontroller then performs calculation to obtain the distance between the vehicles and follow the vehicle. Where MPLAB with $\mathrm{C} 18$ compiler support $\mathrm{C}$ language programming was used for the programming part. The structure of the robot is good but it can travel at a constant speed only. Besides, the following vehicle could not avoid any obstacle or collision [2]. According to [3], a car-like robot equipped with a system called HANS, is able to navigate in an autonomous and safe manner, performing trajectories similar to the ones carried out by human drivers. The system was successfully tested in both simulations and in a laboratory environment using a mobile robot to emulate the carlike vehicle. As a result, this autonomous car can follow the front vehicle in curve road. Besides, this mobile robot also can follow the road, keeping the car in the right lane, maintaining safe distances between vehicles, and avoiding collision. For this mobile robot, it is assumed that there are no cars driving faster than the HANS vehicle which means that no cars will appear from behind. HANS uses a low resolution web camera located in the centre of the vehicle behind the rear-view mirror and a set of sixteen sonar sensor. The key role of the camera is to act as a vision system. It is used to detect the side lines that bound the traffic lanes, the position and orientation of the robot relative to these lines, and the vehicles driving ahead and determining their lane and distance to the robot. Maxelbot leader uses the ultrasonic transmitter to transmit a signal 
through the parabolic cone. The purpose of the parabolic cone is to transmit the signal at 360 degree to the surrounding. The three receivers of the following car will catch the signal transmitted from the transmitter. Then the following car will perform mathematical calculation based on the distance of three receivers from the transmitter. Base on the calculation result, the follower predicts the distance and angle of leader relative to the follower. Limitation of Maxelbot robot is that the effective distance can be measured by the follower from the leader is about one meter only. The advantage of Maxelbot robot is relatively low cost when compared to the vision system base method. Besides, the robot is more robust when performing the following task in multiple obstacles environment compared to following system utilizing IR sensors only [4]. A new robot was constructed to follow human or another robot using the vision system. The vision system is utilized SIFT algorithm. In this algorithm, the robot uses the feature extracted from the training image of target to track the target. Firstly, it uses the SIFT algorithm to recognize the target. After the target is recognized, it estimates the position of the target. Then it uses the PID controller to control the motor to maintain the minimum distance between the follower and the target. There are a number of limitations of this method for following behavior. The 3.5 meter effective distance for the recognition system is adequate for a small robots operating indoors, but would not be adequate for larger outdoor platforms. Additionally, direct pursuit of the leader's current position is quite impropriate and does not work well in complex environments. PID control loops were time consuming to properly tune, and the performance of the simple robot platform limits the applicability of the system as implemented to wider applications. The advantages of this system are the immunity to orientation and occlusion problems made the system easy to use. Also, Object recognition also allows for the implementation of a wide variety of different behaviours based upon a set of different trained objects, opening the way for new avenues of human robot cooperation [5]. Chuong designed leader follower robots to perform validation real time test of follower robot based on leader's trajectory and obstacle avoidance. The Ultrasonic sensor, Infrared sensor and Arduino UNO are the main chosen components. Good results and acceptable accuracy are gained based on straight line, U-shape, S-shape, and Zigzag-shape trajectory in both cases with and without obstacles [6]. Simple basic electronics is used instead of costly microcontrollers which made it very much cost effective. Further modification of this robot includes additional sensors like sonar and infrared so that the robot will be able to follow a line having the ability to avoid obstacle. The robot is designed to follow very tight curves as the data from the sensors are continuous in nature. This robot is simple but effective having straightforward design to perform line following task. Turning angle of the robot depends majority on the speed and motor breaking. Larger turning angle and efficient motor breaking mechanism are required for greater speed. The robot has speed of about 0.2 $\mathrm{m} / \mathrm{s}$. The minimum turning angle is about $110^{\circ}$. The robot missing the line for any angle less than $110^{\circ}[7]$.

The paper is organized as follows. A brief literature review of corporation robots is presented in Section 1. Robot components and circuit design presented Section 2. Testing the motor circuit explained in Section 3. In Section 4 , the experimental results of the robots on a path line following modes and directions are explained. Finally, the concluding remarks are shown in Section 5.

\section{Robot Components}

Design and construction of Mobile Robot requires a broad range of engineering skills such as electronics design, mechanical design, and program design. To build this project we need know how to build the sensor circuit, comparator circuit, controller circuit and motor driver circuit as showing in Figure 1. The mobile robot is divided into two main parts, namely the software and hardware. For the software, PIC16F877A micro controller will be use for system controller for this robot. While on the hardware side, a circuit will be built and connected to sensors and motors. For that case Infrared Light sensor and NPN transistor, are used because of its less much interferences. The control has 6 modes of operation, turn left/right, forward/reverse, and stop. The actual action is caused by controlling the direction/speed of the two motors (the two back wheels), thus causing. Two motors as a output will control by motor driver that connected to the PIC16F877A.

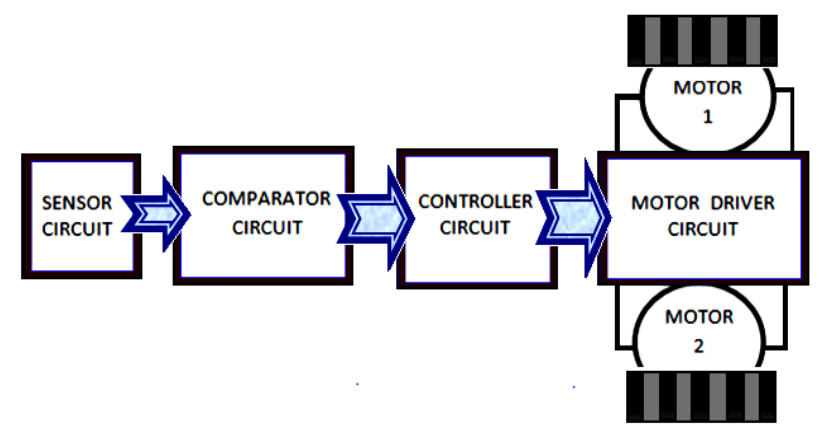

Figure 1. Block diagram of robot design.

\subsection{IR Sensor}

In this paper, mobile robot uses 4 IR LEDs (Tx) and 4 IR sensors $(\mathrm{Rx})$ with distance between the two sensors is $15 \mathrm{~mm}$. the first $\mathrm{Rx}$ receives an analog signal that depends on the intensity of light reflected by the black line of emitted beam by the Tx. These signals are sent to the comparator LM324 with creates digital signals ( 0 or 1$)$ that are sent to microcontroller PIC.

Hence, the distance between sensors and ground surface is important and it is more important that how to put 
sensors near each other. The distance between sensors and ground surface must be 2 to $10 \mathrm{~mm}$ and the distance between each sensor is depending on the line width. Mobil robot used four pair of sensors as shown in Table 1.

Table1. Sensor Arrays.

\begin{tabular}{|l|l|l|l|}
\hline Tx1(left) & Tx2(center) & Tx3(center) & Tx4(right) \\
\hline $\mathrm{Rx} 1$ (left) & $\mathrm{R} \times 2$ (center) & $\mathrm{R} \times 3$ (center) & $\mathrm{R} \times 4$ (right) \\
\hline
\end{tabular}
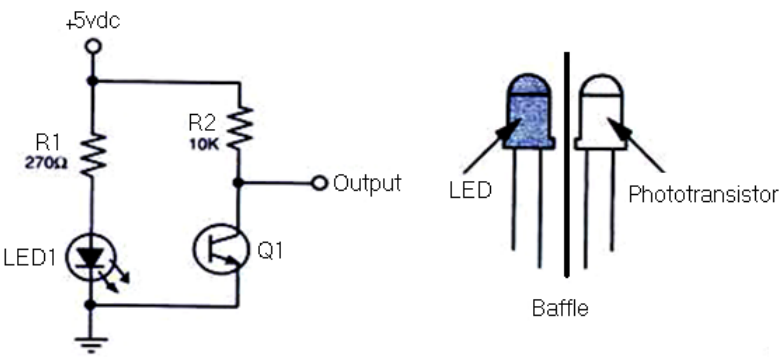

Figure 2. The basic design of IR sensor.

IR LED emits infrared radiation. This radiation illuminates the surface in front of LED. Surface reflects the infrared light. Depending on reflectivity of the surface, amount of light reflected varies. This reflected light is made incident on reverse biased IR sensor. When photons are incident on reverse biased junction of this diode, electron-hole pairs are generated, which results in reverse leakage current. Amount of electron-hole pairs generated depends on intensity of incident IR radiation. More intense radiation results in more reverse leakage current. This current can be passed through a resistor so as to get proportional voltage. Thus as intensity of incident rays varies, voltage across resistor will vary accordingly.

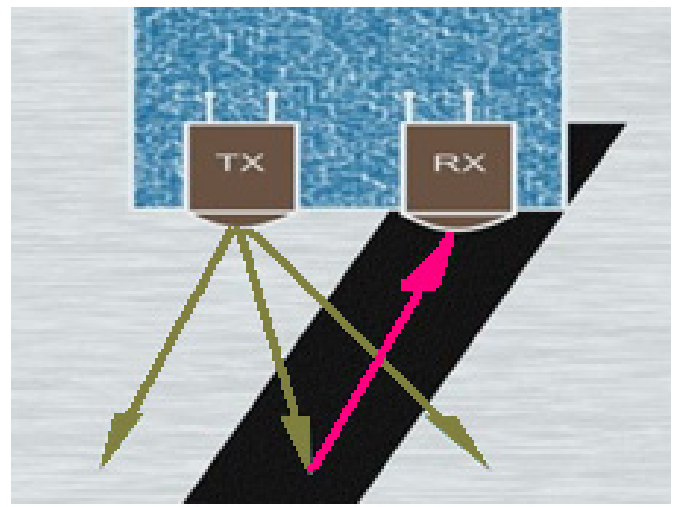

Figure 3. Infrared reflection.

\subsection{Comparator}

The received signals from the sensors are analog and converted to the digital form. Therefore, the circuit can be designed to send the sensors signals to the processor directly. Hence, the processing time can be managed just by using an external comparator. LM324 is a good comparator that is used in this paper. One LM324 can support four sensors.

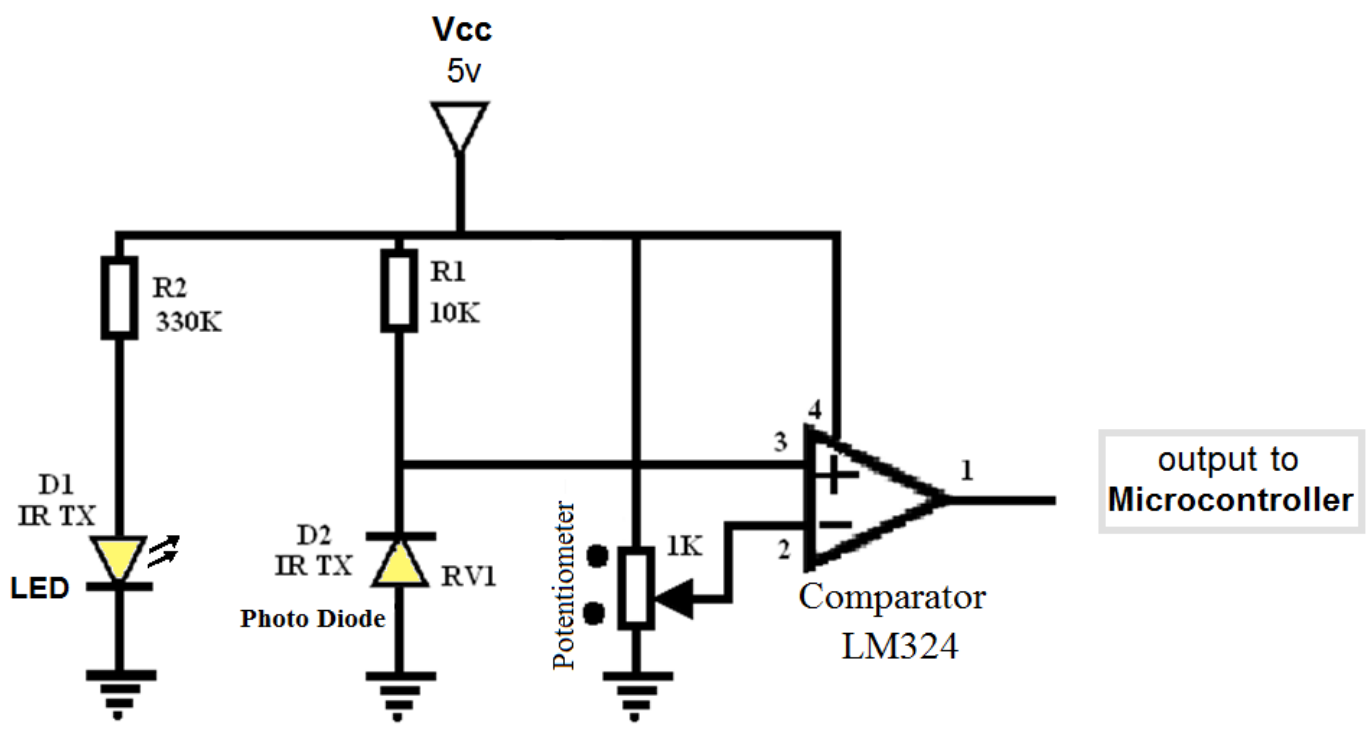

Figure 4. Schematic of comparator and IR sensor circuit 


\subsection{Microcontroller}

In this study, PIC16F877A was used as a processor. PIC16f877A is a good flash microcontroller that has many features a 40-pin and 5 input-output (I/O) ports. This flash microcontroller may be programmed and erased instantly, without the need for a UV light sours, to speed program testing. Reprogram ability offer a highly flexible solution today's ever- changing market demands, where product updates and modification are routinely carried out in the field. Port A consists of 6 pins which can be set up as either digital $\mathrm{I} / \mathrm{O}$ or analog inputs. Port $\mathrm{B}$ is an 8 pin port which can be used for both digital $\mathrm{I} / \mathrm{O}$ operations and in-circuit debugger (ICD) operations. Port $\mathrm{C}$, on the other hand, is a 5-pin multi-functional port, which can be used for digital $\mathrm{I} / \mathrm{O}$, as capture-compare (CCP) input, or pulse-width modulation (PWM) output. Port D is an 8-pin port, which can be used for both digital $\mathrm{I} / \mathrm{O}$ as well as parallel slave port (PSP) functions. Finally, port Eis a 3-pin port, which is used for external memory connections.

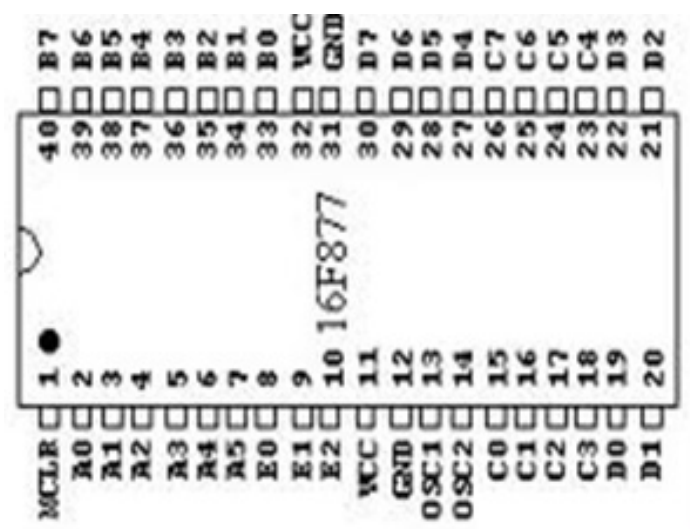

Figure 5. Microprocessor Unit

\subsection{Driver Motor}

This paper needs a driver IC controlling and giving power to the motor. The microcontroller sends a signal to the driver which acts as a switch. The microcontroller only sends signal to the switch and then the switch must gives required voltage to the motor. If the received signal by the driver is high, it will rotate the motor.

This robot used two motor drives MD30B. It was capable of driving up to $30 \mathrm{amp}$ peak motor current. Added with extra LED status indicators and better protection, it become more user friendly and more reliable, simple connect the power source, this driver is ready to drive high current motor. It has been designed with capabilities and features as followed:

- Drive up to 20 amp continuously and peak $30 \mathrm{amp}$ for a few seconds.

- $\quad 5 \mathrm{~V}$ logic level inputs.

- $\quad$ PWM speed control up to $10 \mathrm{KHz}$

- Bi-directional control of 1 motor.

- 2 on-board push buttons for fast testing.

- Onboard PWM generation
- Heat sink with fan for fast thermal release.

- 2 LEDs as output indicator.

- 1 LED as Vin indicator

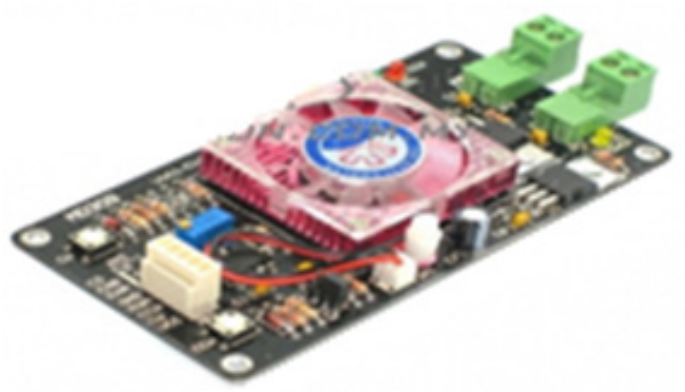

Figure 6. Driver motor MD30B

The microcontroller sends instruction to the driver after processing the data coming from sensors part. The driver gives positive voltage to one of the motor pins and gives negative voltage to another one which is provide five states:

- Move forward - M1\&M2 ON (rotate forward).

- Move right - M1 ON.

- Move left - M2 ON.

- $\quad$ Move backward - M1\&M2 ON (rotate backward).

- $\quad$ Stop - M1\&M2 OFF.

\subsection{Motor}

DC motor is the most compatible motor used to generate this type of robot. The output from the motor will be combined with control circuit. The DC motor is built by a loop of wire that set on rotated shaft situated in between everlasting magnet. When current is applied on a conductor, a spherical magnetic flux will be produces around the conductor. The left hand rule is applied in determine the direction of resulting flux line. The function of DC motor is to convert from electrical power to mechanical power. The converted power is produces by the applied current from the loop and then forming the magnetic flux to move the motor. The robot used two DC motors that specification as follows:

- DC $12 \mathrm{v}$.

- Output power 1.1 Watt.

- $\quad$ Rated speed 185 RPM.

- $\quad$ Rated current $410 \mathrm{~mA}$.

- $\quad$ Rated torque 78.4 mN.m

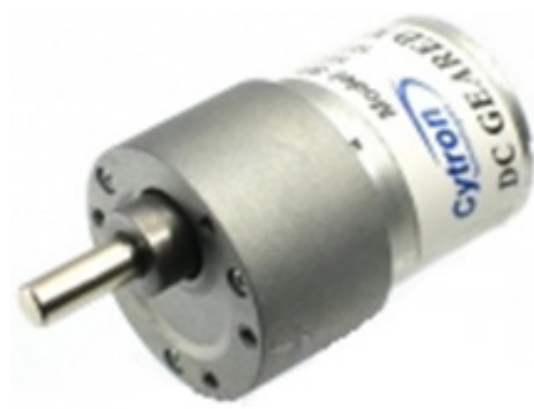

Figure 7. DC Motor 


\subsection{LM7805 Voltage Regulator}

The LM7805 as shown in figure 8 is positive regulators are available in the TO-220package and with several fixed output voltages making them useful in a wide range of applications. For this paper, the $5 \mathrm{~V}$ fixed voltage is used to supply voltage to the microcontroller (PIC16877A), sensor circuit and comparator circuit. Each type employs an internal current limiting, thermal shut down and safe operation area protection. This LM7805 is to protect the circuit. It function is to limit the voltage that flow through it before go to circuit like microcontroller. it can minimize the voltage, thus LM7805 fixed the voltage to $5 \mathrm{~V}$ the input power supply to LM7805 come from 9V battery. The features LM7805 is like output current up to 1A. For output voltages are $5 \mathrm{~V}, 6 \mathrm{~V}, 10 \mathrm{~V}, 12 \mathrm{~V}, 18 \mathrm{~V}$, and $24 \mathrm{~V}$. Than it provides with thermal protection, short circuit protection and also output transistor safe operating area protection.

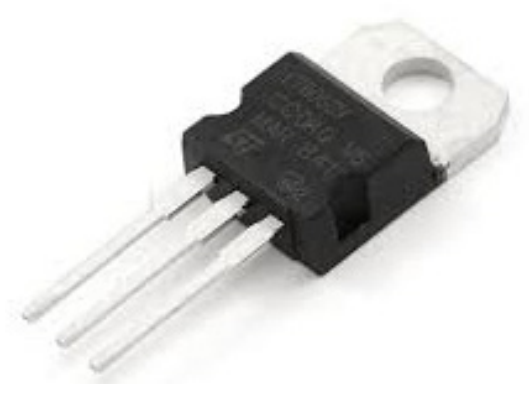

Figure 8. LM7805 Voltage Regulator

\section{Hardware Testing and Analysis}

In this section, we present experiments results for testing motor circuit and motor circuit measurement voltage.

\subsection{Testing the Motor Circuit}

The mobile robot consists of four pair of infrared sensors that are used as inputs and two DC motor as outputs. Each combination of sensors will give a different product and will determine the direction movement of the robot. Tables 2,3 shows the motor testing of mobile robot.

Table 2. Motor circuit testing when robot follows white line.

\begin{tabular}{|c|c|}
\hline $\mathrm{Rx} 1 . \mathrm{Rx} 2 . \mathrm{R} \times 3 . \mathrm{Rx} 4$ & Action of Robot \\
\hline 1111 & Stop \\
\hline $\begin{array}{llll}1 & 0 & 0 & 1 \\
1 & 0 & 1 & 1 \\
1 & 1 & 0 & 1\end{array}$ & Forward \\
\hline $\begin{array}{lllll} & 0 & 1 & 1 \\
0 & 1 & 1 & 1 \\
\end{array}$ & Turn Right \\
\hline $\begin{array}{llll}1 & 1 & 0 & 0 \\
1 & 1 & 1 & 0\end{array}$ & Turn Left \\
\hline
\end{tabular}

Table 3. Motor circuit testing when robot follows black line.

\begin{tabular}{|c|c|}
\hline $\mathrm{Rx} 1 . \mathrm{Rx} 2 . \mathrm{Rx} 3 . \mathrm{Rx} 4$ & Action of Robot \\
\hline 0000 & Stop \\
\hline $\begin{array}{lllll} & 1 & 10 \\
0 & 10 & 0 \\
0 & 0 & 1 & 0 \\
\end{array}$ & Forward \\
\hline $\begin{array}{llll}1 & 1 & 0 & 0 \\
1 & 0 & 0 & 0 \\
\end{array}$ & Turn Right \\
\hline $\begin{array}{lllll}0 & 0 & 1 & 1 \\
0 & 0 & 0 & 1\end{array}$ & Turn Left \\
\hline
\end{tabular}

\subsection{Motor Circuit Analysis}

The motor circuit analysis is to measure the voltage and the speed of motor.

The motors are working perfectly as the analyzed tables 2-5. The approach to line tracking is usually considered in the digital domain where the outputs from the digital line sensors are given to a microcontroller which is programmed to run the motors of the robot depending on where the robot is positioned with respect to the centre of the line.

Table 4. Motor circuit measurement voltage when follow white line.

\begin{tabular}{|c|c|c|c|c|c|c|}
\hline \multicolumn{4}{|c|}{ Input (volt ) } & \multicolumn{2}{|c|}{ Output (volt ) } & \multirow{2}{*}{ Action } \\
\hline $\mathrm{S} 1$ & $\mathrm{~S} 2$ & $\mathrm{~S} 3$ & $\mathrm{~S} 4$ & Motor 1 & Motor 2 & \\
\hline 3.87 & 3.87 & 3.87 & 3.87 & 0 & 0 & Stop \\
\hline 3.87 & 0 & 0 & 3.87 & 10 & 10 & Forward \\
\hline 3.87 & 0 & 3.87 & 3.87 & 10 & 10 & Forward \\
\hline 3.87 & 3.87 & 0 & 3.87 & 10 & 10 & Forward \\
\hline 0 & 0 & 3.87 & 3.87 & 10 & 0 & Turn right \\
\hline 0 & 3.87 & 3.87 & 3.87 & 10 & 0 & Turn right \\
\hline 3.87 & 3.87 & 0 & 0 & 0 & 10 & Turn left \\
\hline 3.87 & 3.87 & 3.87 & 0 & 0 & 10 & Turn left \\
\hline
\end{tabular}


Table 5. Motor circuit measurement voltage when follow black line.

\begin{tabular}{|c|c|c|c|c|c|c|}
\hline \multicolumn{4}{|c|}{ Input (volt ) } & \multicolumn{2}{|c|}{ Output (volt ) } & \multirow[t]{2}{*}{ Action } \\
\hline $\mathrm{S} 1$ & $\mathrm{~S} 2$ & $\mathrm{~S} 3$ & $\mathrm{~S} 4$ & Motor 1 & Motor 2 & \\
\hline 0 & 0 & 0 & 0 & 0 & 0 & Stop \\
\hline 0 & 3.87 & 3.87 & 0 & 10 & 10 & Forward \\
\hline 0 & 3.87 & 0 & 0 & 10 & 10 & Forward \\
\hline 0 & 0 & 3.87 & 0 & 10 & 10 & Forward \\
\hline 3.87 & 3.87 & 0 & 0 & 10 & 0 & Turn right \\
\hline 3.87 & 0 & 0 & 0 & 10 & 0 & Turn right \\
\hline 0 & 0 & 0 & 3.87 & 3.87 & 10 & Turn left \\
\hline 0 & 0 & 0 & 3.87 & 0 & 10 & Turn left \\
\hline
\end{tabular}

\subsection{Output Waveform}

To get the output waveform from the circuit we use the oscilloscope. From this equipment we will get the measurement value and waveform.

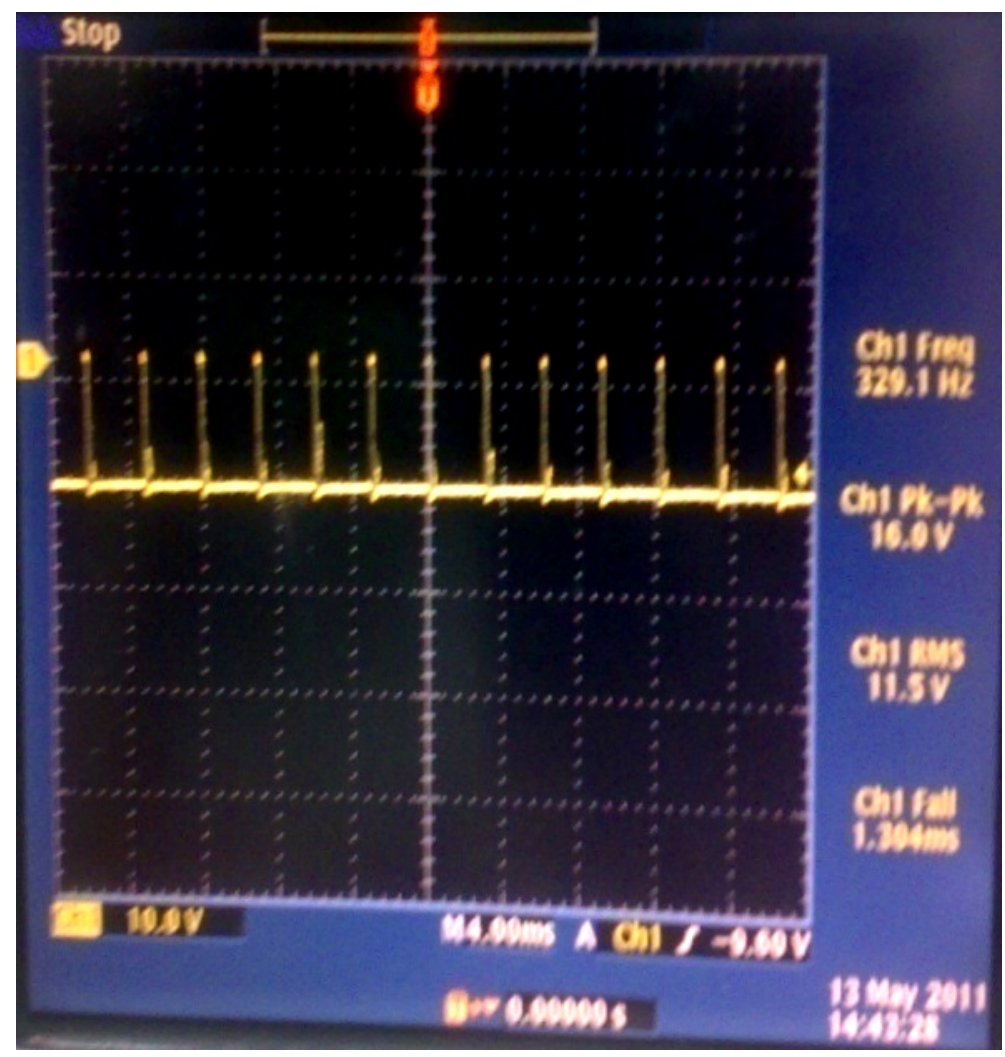

Figure 9. Waveform of output voltage at the motor driver when move forward. 


\section{Flowchart Programming and System Implementation}

The movement systems are important part of a robot and it is objective is how to move robot from one point to another point. These robots designed with five modes of operation, turn left/right, forward/reverse, and stop. To move forward, both of the motors are turn on and rotate forward simultaneously. For the turning right, the left motor is turn on and right motor is turn off and vice versa for turning left. The outer sensors are the most important for the line tracking. The robot supposes that it is placed on the line and that it is running forward. The outer sensors capture the dark colour. If not, the left sensor captures the dark colour, then the robot is turns left (the line got under the sensor because of the line curve or because the robot is turning too much to the right) and it keeps turning until the left sensor captures the dark colour until the line got in between the sensors again. Figure 10 shows the flow chart of programming development of line following robot.

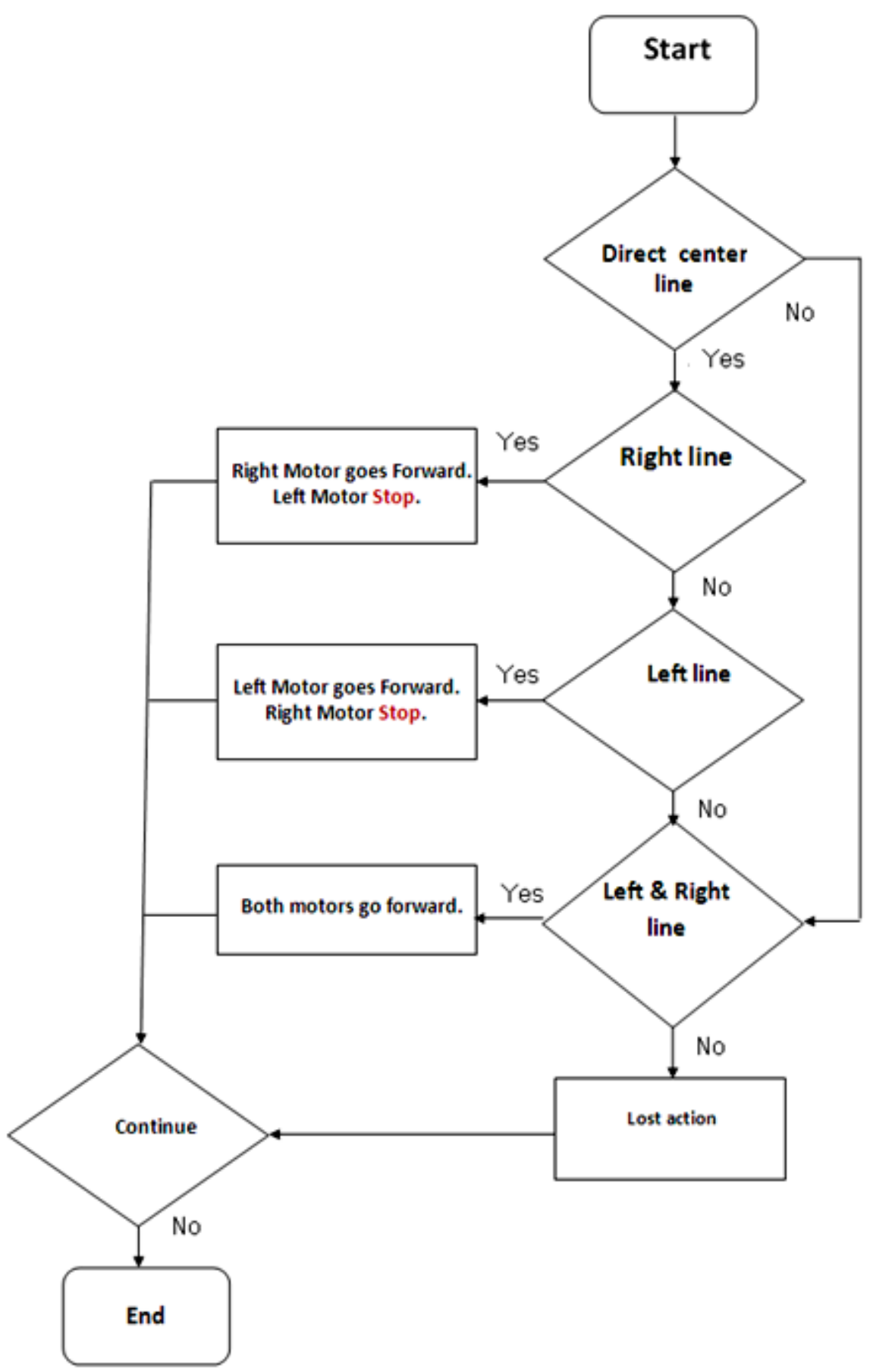

Figure 10. Flow chart of programming development for line following robot. 
The final results for line following robots carrying load shown in Figures 11-13 below, where the line and carry load, both of robots travelling at same speed.

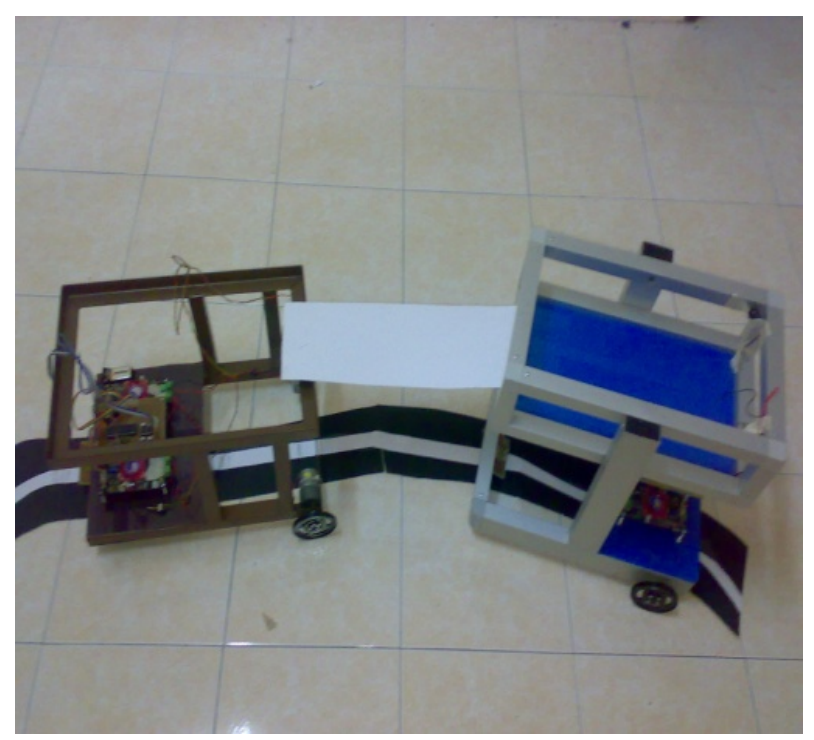

Figure 11. The Robots follow the line and carry the load.

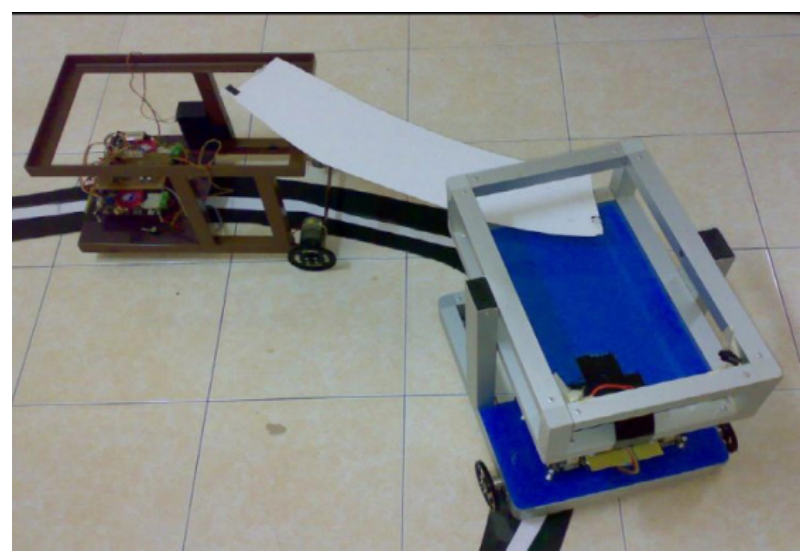

Figure 12. Line following robots turn left and carrying load.

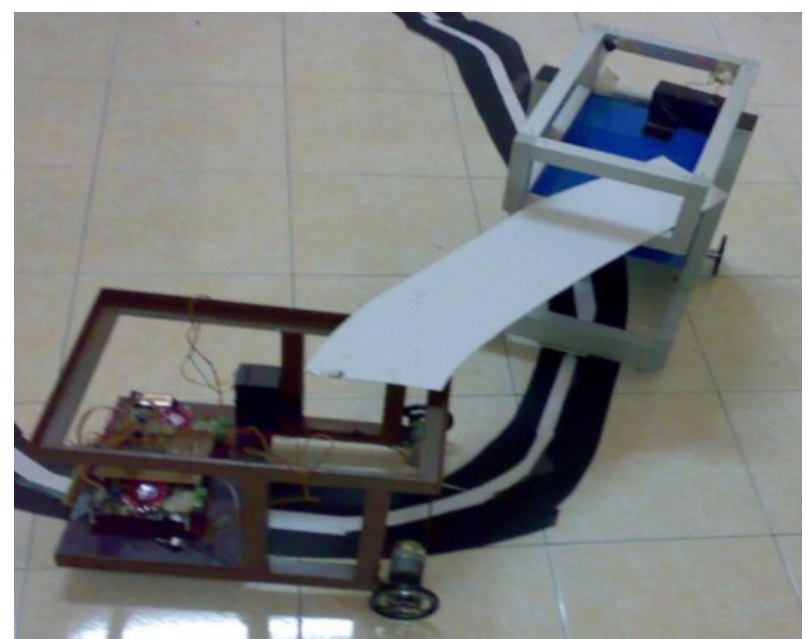

Figure 13. Line following robots turn right and carrying load.
These experiments worked successfully based the robots five modes of operation, turn left/right, forward/reverse, and stop. Both robots are experimentally stable and hence the robots are able to take advantage of smooth handling the objective. A Single robot has a limitation on handle load capacity such as cannot handle heavy load and long size load. To overcome this limitation an easier way is to have a groups of mobile robots working together to accomplish an aim that no single robot can do alone. As a result the robots are built-up successfully as per their functionality. This kid of robots can be used for many applications such as smart medical trolley and cart luggage trolley.

\section{Conclusions}

In this paper two following line robots have been built. Both of the robots follow the line and work together as leader and slave to carry load from one point to another point. The simple construction and cost efficient are significant features of this project. The robots are built-up successfully as per their functionality. The experiments demonstrated that robots are able to take advantage of carrying the load with accurate behavior.

\section{REFERENCES}

[1] G. Lefranc. Colony of robots: New Challenge, Workshop invited key lecture, 2008.

[2] G. H. Wen. Development of a road vehicle model for road vehicle convoy System, UTM thesis, 2007.

[3] J. Sequeira., A. Goncalves., A. Godinho. Low cost Sensing for Autonomous Car Driving in Highways. ICINC0-RA (2), 370-377, 2007.

[4] Rozan Boudville. Obstacle detection and avoidance mobile robot. UTM thesis, 2004.

[5] J. Giesbrecht., Leader/Follower Behaviour Using the SIFT Algorithm for Object Recognition. Defence Research and Development Canada, Technical memorandum TM 2006-108, 2006.

[6] L. Chuong, leader follower robot, UTeM Malaysia, Thesis, 2015.

[7] K. Hasan,. A. Naid., A. Mamun. Implementation of Autonomous Line Follower Robot. IEEE/OSA/IAPR International Conference on Informatics, Electronics \& Vision, 865-869, 2012. 